Revista de Metalurgia

Vol 53, Número 1, Enero-Marzo 2017, e087

ISSN-L: 0034-8570

http://dx.doi.org/10.3989/revmetalm.087

\title{
NOTA TÉCNICA \\ Efecto del reprensado en las propiedades mecánicas de guías de válvulas fabricadas por pulvimetalurgia
}

\author{
Andrés Rodríguez-Spitia ${ }^{\mathrm{a}}$, Gustavo Agudelo-Ospina ${ }^{\mathrm{a}}$, Juan Galindo ${ }^{\mathrm{a}}$, Faber Correa-Ballesteros ${ }^{\mathrm{a}, \mathrm{b}}$, \\ Nelly Alba de Sánchez ${ }^{\mathrm{a}, \mathrm{b} \varpi}$ \\ ${ }^{a}$ Grupo Ciencia e Ingeniería de Materiales, Universidad Autónoma de Occidente, \\ Autopista Cali Jamundi \#Cll 25, Valle del Cauca, Cali, Colombia

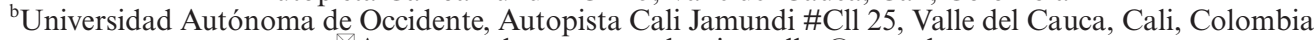 \\ Autor para la correspondencia: nalba@uao.edu.co
}

RESUMEN: Se determinaron la dureza, pérdida de masa y coeficiente de fricción, de guías de válvulas para motores de combustión interna fabricadas en latón por proceso de pulvimetalurgia, con el objetivo de conocer si cumplen con las exigencias que requiere un motor de combustión interna, utilizando como parámetro comparativo guías de válvulas comerciales de latón producidas por fundición. Se estudió, si las propiedades son homogéneas a lo largo de toda la guía de la válvula y se evaluó el proceso de reprensado que se lleva a cabo de manera adicional en la línea de producción. Se determinaron las durezas Brinell y Vickers; el coeficiente de fricción y la tasa de desgaste (ensayo pin on disk). Mediante microscopía electrónica de barrido y análisis metalográfico, se estudió el mecanismo de desgaste. Se llevó a cabo la determinación de la composición química de las guías y el efecto del reprensado cuantificando la porosidad superficial, mediante el software Analizador de imágenes Pro Plus ${ }^{\circledR}$. Se puso de manifiesto que las piezas fabricadas por proceso de pulvimetalurgia presentaron valores inferiores en sus propiedades mecánico-tribológicas en comparación con las guías de válvula comerciales. Estos resultados permitirán mejorar las condiciones de fabricación de las guías mediante pulvimetalurgia.

PALABRAS CLAVE: Coeficiente de fricción; Dureza; Efecto de reprensado; Pérdida de masa

Citar como/Citation: Rodríguez-Spitia, A.; Agudelo-Ospina, G.; Galindo, J.; Correa-Ballesteros, F.; Alba de Sánchez, N. (2017) "Efecto del reprensado en las propiedades mecánicas de guías de válvulas fabricadas por pulvimetalurgia”. Rev. Metal. 53(1): e087. http://dx.doi.org/10.3989/revmetalm.087

\begin{abstract}
Effect of repressing on the mechanical properties of the valve guides manufactured by powder metallurgy. Characterization of hardness, mass loss, and friction coefficient, was carried out on valve guides for internal combustion engines, manufactured by powder metallurgy, with the aim of finding out if they fulfill the requirements of an internal combustion engine and to be mass produced. Currently available brass casting valve guides were used as comparative parameter. It was analyzed whether properties were present in a homogeneous way in the piece lengthwise; the additional repressing process carried out in the production line with respect to the sintered guides was evaluated. Therefore, Brinell, Vickers and dry slip pin-on-disk tests were done in order to determine the friction coefficient and the wear rate. Scanning electron microscopy SEM and the metallographic analysis were used to study the wear mechanism, chemical composition of the guides, and the effect of repressing -quantifying the porosity. It was clear that these pieces manufactured by powder metallurgy process showed
\end{abstract}


lower values in their mechanical-tribological properties compared to commercially available valve guides taken as reference. With these results, the company which manufactures the guides by powder metallurgy will make some adjustments suggested for the parameters at the guide's production line.

KEYWORDS: Friction coefficient; Hardness; Mass loss; Repressing effect

ORCID: Andrés Rodríguez-Spitia (http://orcid.org/0000-0001-9634-9626); Gustavo Agudelo-Ospina (http://orcid. org/0000-0002-9490-3627); Juan Galindo (http://orcid.org/0000-0002-7105-3367); Faber Correa-Ballesteros (http:// orcid.org/0000-0002-2954-2853); Nelly Alba de Sánchez (http://orcid.org/0000-0002-9225-8046)

Copyright: (C) 2017 CSIC. Este es un artículo de acceso abierto distribuido bajo los términos de la licencia Creative Commons Attribution (CC BY) España 3.0.

\section{INTRODUCCIÓN}

Hoy en día, el negocio de venta de piezas de automóvil es un mercado altamente competitivo, a nivel mundial (ProExport, 2012). Las empresas fabricante, buscan producir estas piezas en gran cantidad y en el menor tiempo posible para reducir costos de fabricación y comercialización. Actualmente, se está implementando en las líneas de producción de componentes de automóvil, el proceso de pulvimetalurgia $(P M)$ ya que ofrece varias ventajas con respecto a la fabricación tradicional por fundición. Sin embargo, el valor de la materia prima en polvo es alto (Groover, 1997). Algunas de las ventajas del proceso por pulvimetalurgia para el conformado de piezas es la reducción y en algunos casos, la eliminación del mecanizado, ahorro de material o materia prima con la facilidad de producir piezas en serie (Groover, 1997; De Garmo et al., 2002; Schmid y Kalpakjian, 2002), y también, la característica de ser piezas auto lubricadas (Martín et al., 2015). Una de estas piezas de automóvil que se producen por fundición, son las guías de válvulas (GV) para los motores de combustión interna, que actualmente se fabrican por PM y que tienen como función, guiar el desplazamiento longitudinal de la válvula de apertura o cierre y disipar el calor absorbido por las válvulas, como consecuencia del proceso de combustión. Por ello, estas piezas se fabrican con materiales que poseen alta conductividad térmica como aleaciones de cobre (Dietsche, 2005; Gonzales Calleja, 2015).

En estudios similares realizados anteriormente, Jang et al. (2000) determinaron que algunas piezas fabricadas por PM que presentan alta resistencia, pueden sustituir a piezas elaboradas por forja, comprobándose mediante la elaboración de un disco de clutch para automóvil. Adicionalmente, Fujiki (2001) indicó que esta tecnología puede ser usada ampliamente en el sector automovilístico debido a sus ventajas frente a la tecnología tradicional. Por otro lado, Abdizadeh et al. (2014) realizaron el estudio de la microestructura y propiedades mecánicas de un compuesto reforzado con $\mathrm{MgO}$, fabricado por fundición y metalurgia de polvos, donde se puso de manifiesto que el método de fundición presenta mayor homogeneidad y valores más altos de las propiedades mecánicas en comparación con la PM, debido a la baja porosidad presente en el material.

Una empresa de la ciudad de Cali, Colombia, en proceso de desarrollo de producción de GV para motores de combustión interna fabricadas en latón mediante proceso de PM, vio la necesidad de caracterizar las guías con proceso de sinterizado y reprensado (proceso adicional para mejorar las dimensiones finales a las piezas (Mott, 2006) para competir con el mercado internacional; los resultados se compararon con guías fabricadas mediante proceso de fundición que se encuentran en el comercio. En esta investigación se realizó la caracterización de dureza, coeficiente de fricción y pérdida de masa de las GV. Con los resultados obtenidos, se pueden introducir mejoras en el proceso industrial de producción de válvulas.

\section{MATERIALES Y MÉTODOS}

\subsection{Materiales}

Las GV analizadas en esta investigación son producidas con aleación de cobre y zinc (latón) como base; sin embargo, las fabricadas por proceso de PM contienen otros elementos adicionales como se presenta en la Tabla 1, a diferencia de las $\mathrm{GV}$ producidas mediante fundición. Estos elementos de aleación han permitido mantener las dimensiones de la guía en el momento de su montaje. Se analizaron dos tipos de válvulas de diferentes longitudes (47 y $49 \mathrm{~mm}$ ) identificadas como L47 y L49 que se seccionaron en tres (3) zonas; en la Fig. 1 se presenta una imagen de una GV donde se identifica la zona superior con la letra A, la zona central con la letra B y la zona inferior con la letra C; este seccionamiento permitirá determinar si existe homogeneidad de las propiedades a lo largo de su longitud.

TABLA 1. Elementos químicos presentes en GV fabricadas por proceso de PM

\begin{tabular}{lllll}
\hline Material & \multicolumn{4}{c}{ Elementos químicos } \\
\hline Latón & $\mathrm{Cu}$ & $\mathrm{Zn}$ & $\mathrm{Mn}$ & $\mathrm{Fe}$ \\
\hline
\end{tabular}


(A)

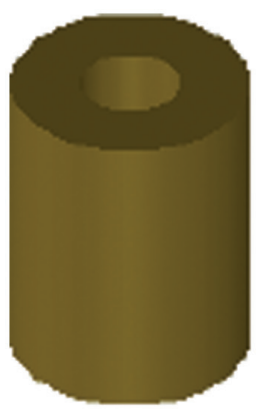

(B)

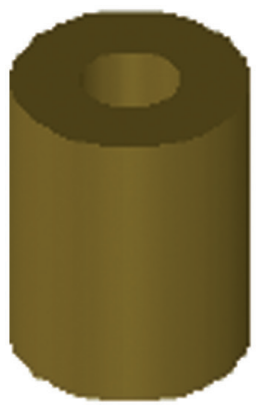

(C)

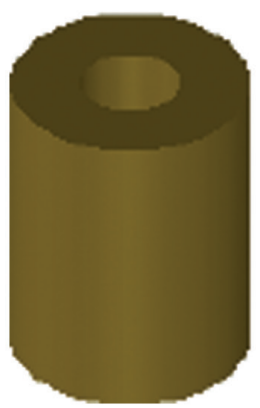

Figure 1. Guía de válvula completa y seccionada.

\subsection{Ensayos}

\subsubsection{Caracterización de Dureza}

Para realizar las caracterizaciones de dureza Brinell y Vickers a las GV se utilizaron las normas ASTM E10-12 (2012) y ASTM E92-82 (2003), respectivamente, donde se indentó cinco veces la sección transversal para obtener el valor promedio de esta propiedad. Se empleó el durómetro AFFRI modelo 206 EX para la toma de dureza Brinell y el durómetro Zwick/Roell indentec ZHV1 para la caracterización de micro dureza. Los parámetros empleados se muestran en la Tabla 2 y Tabla 3 para la toma de macro dureza y micro dureza, respectivamente.

\subsubsection{Prueba de Desgaste}

Para la caracterización del coeficiente de fricción en GV se realizó ensayo de pin on disk empleando el tribómetro Microtest modelo MT/10/SCM bajo la
TABLA 2. Parámetros para ensayo dureza Brinell

\begin{tabular}{lcc}
\hline Diámetro Balín D $(\mathbf{m m})$ & Carga $\mathbf{P}(\mathbf{N})$ & P/D $^{\mathbf{2}}$ \\
\hline 2,5 & 613 & 10 \\
\hline
\end{tabular}

TABLA 3. Parámetros para ensayo dureza Vickers

\begin{tabular}{lcc}
\hline Carga (kgf) & Tiempo de Sostenimiento & Indentador \\
\hline 0,5 & 20 & Diamante piramidal \\
\hline
\end{tabular}

TABla 4. Parámetros de ensayo pin on disk

\begin{tabular}{lc}
\hline Datos & Valores \\
\hline Recorrido (m) & 1000 \\
Velocidad angular (rpm) & 200 \\
Carga normal (N) & 2 \\
Radio de pista (mm) & 5 \\
\hline
\end{tabular}

TABLA 5. Dureza Brinell de guías de válvulas

\begin{tabular}{lccc}
\hline & \multicolumn{3}{c}{ Dureza Brinell (HB) } \\
\cline { 2 - 4 } & Zona & Sin reprensar & Reprensada \\
\hline L47 & A & 37 & 54 \\
& B & 34 & 50 \\
L49 & C & 38 & 68 \\
& A & 35 & 51 \\
& B & 34 & 42 \\
& C & 40 & 81 \\
\hline
\end{tabular}

norma ASTM G99-05 (2000). La selección de parámetros de ensayo se presenta en la Tabla 4. Antes y después del ensayo se pesaron las probetas en una balanza con precisión de $10^{-4} \mathrm{~g}$; para determinar la pérdida de masa ocasionada por el desgaste del balín sobre la probeta.

\section{RESULTADOS Y ANÁLISIS}

\subsection{Dureza Brinell y Vickers}

Los valores promedio de dureza Brinell para las GV L47 y L49 sin reprensar y reprensadas se presentan en la Tabla 5. La zona central B presentó la menor dureza y la zona inferior $\mathrm{C}$ el mayor valor. Se aprecia que con proceso de reprensado se incrementó el valor de dureza.

Las GV con proceso de sinterizado presentaron alta homogeneidad en la dureza a lo largo de la longitud, mientras que con proceso adicional de reprensado no se evidenció este comportamiento; pero sí un incremento de esta propiedad debido a la deformación plástica inducida a la guía de válvula en el momento de su producción y a la disminución de poros, ya que para llevar a cabo el proceso de 
reprensado se emplean cargas de compactación elevadas que logran aumentar la densidad de la pieza, lo que equivale a menor porosidad y mayor dureza. Los resultados anteriores son coherentes con los alcanzados en un estudio anterior realizado por Keraghel et al. (2011).

La baja homogeneidad en la dureza en GV con proceso de reprensado se debe a que los dos punzones de la prensa empleada para este proceso adicional no ejercen igual carga sobre el material; mientras que en las guías con solo proceso de sinterizado si ejercen la misma carga lo que está relacionado con la configuración de la prensa utilizada para la compactación del material.

Las zonas centrales B presentaron menor dureza debido a que el polvo metálico se compacta de manera vertical en el proceso de prensado, y esta zona es la más alejada de los punzones que ejercen la fuerza axial hacia el centro de la guía desde la zona superior A y zona inferior C; generando que esta zona sea de menor densidad, debido a la alta porosidad, cuantificada con el uso de un analizador de imágenes Pro Plus, con respecto a las zonas A y C. En la Fig. 2 se presentan los valores de la porosidad superficial en las diferentes zonas de las GV.
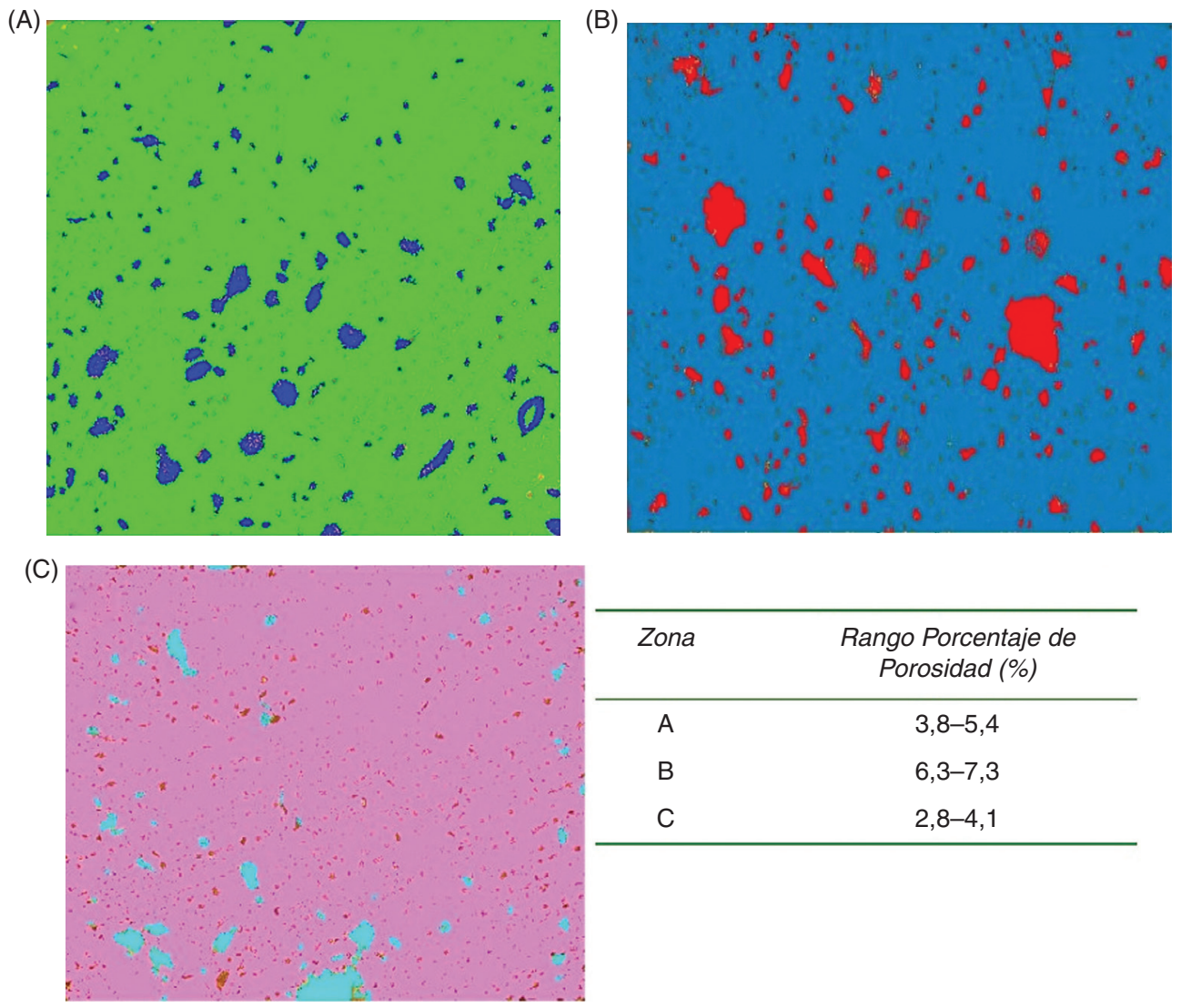

FiguRE 2. Rango de porcentaje de porosidad en guía de válvula L47 sin reprensar.
(B)

\begin{tabular}{cc}
\hline Zona & $\begin{array}{c}\text { Rango Porcentaje de } \\
\text { Porosidad (\%) }\end{array}$ \\
\hline A & $3,8-5,4$ \\
B & $6,3-7,3$ \\
C & $2,8-4,1$ \\
\hline
\end{tabular}

La comparación de la dureza Brinell entre las GV reprensadas y comercial se muestra en la Fig. 3. Las GV comerciales presentaron mayor dureza en comparación con las GV fabricadas por PM.

La diferencia en las durezas, de las GV de acuerdo a la Fig. 3, fabricadas por PM y por fundición (comerciales), se debe al tipo de proceso de fabricación y los elementos químicos que contienen. La dureza de las GV fabricadas por PM no es apropiada para procesos de mecanizado que proporcionan las dimensiones finales debido a su bajo valor, ya que el material no se desprende en forma de viruta debido a su alta plasticidad, ocasionando tiempos extensos de producción. Estos resultados fueron comprobados en ensayos industriales.

Los valores de dureza Vickers obtenidos para las GV L47 y L49 sin reprensar y reprensadas presentaron comportamiento diferente en comparación con los resultados de dureza Brinell (Tabla 6). No se aprecia homogeneidad de esta propiedad a lo largo de la guía de válvula.

La dureza Vickers en las GV fabricadas por PM no presentaron el mismo comportamiento que la dureza Brinell, debido a que los valores de micro dureza se vieron afectados por la porosidad 


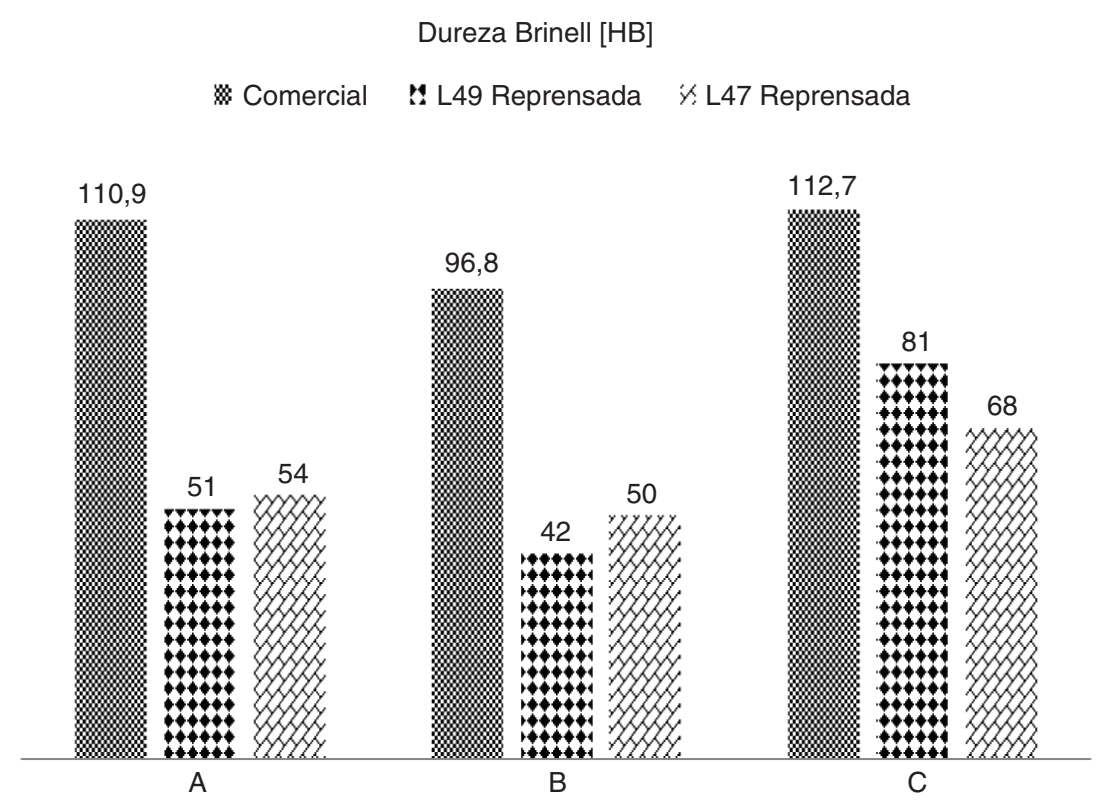

Figure 3. Comparación de dureza Brinell.

TABla 6. Valores de dureza Vickers de guías de válvulas

\begin{tabular}{lccc}
\hline & \multicolumn{3}{c}{ Dureza Vickers (HV) } \\
\cline { 2 - 4 } & Zona & Sin reprensar & Reprensada \\
\hline L47 & A & 66 & 71 \\
& B & 58 & 67 \\
& C & 89 & 108 \\
L49 & A & 55 & 78 \\
& B & 64 & 74 \\
& C & 64 & 118
\end{tabular}

del material que es característico en piezas fabricadas mediante proceso de PM y por la baja homogeneidad de la composición química base del material, donde se evidenció en esta última, zonas con diferentes cantidades de cobre (Fig. 4.) ocasionadas por la pérdida de zinc en el proceso de sinterización.

\subsection{Coeficiente de fricción y pérdida de masa}

Los valores de coeficiente de fricción para las GV fabricadas por proceso de PM y fundición se presentan en la Tabla 7. Se realizó ensayo de pin on disk a la zona A de guía de válvula comercial ya que por ser fabricada por proceso de fundición sus propiedades son similares a lo largo de toda su dimensión (Abdizadeh et al., 2014).

Se apreció baja homogeneidad de esta propiedad a lo largo de su longitud para las GV fabricadas por
PM. El coeficiente de fricción obtenido para la guía comercial fue menor en comparación con los valores de coeficiente de fricción adquiridos para las GV fabricadas por PM.

La baja homogeneidad presentada a lo largo de la longitud de las guías fabricadas por PM, se debió a que esta propiedad se vio afectada por la baja calidad superficial de estas piezas debido a las porosidades presentes en el producto final, ver Fig. 2. El coeficiente de fricción para $\mathrm{GV}$ fabricadas por proceso de fundición fue menor como se esperaba, por ser estas piezas obtenidas de metal en volumen, la presencia de poros que afectan la rugosidad superficial se reducen o se eliminan en algunos casos. Sin embargo, estos valores se presentaron por debajo del valor estándar de un contacto acero-latón $\left(<0,44 \mu_{\mathrm{k}}\right)$.

La pérdida de masa de las GV fue despreciable, lo que indica que presentan alta resistencia al desgaste, propiedad importante para su desempeño; en la Tabla 8 se presentan los resultados.

\subsection{Mecanismo de desgaste}

La baja pérdida de masa de las GV está relacionada con su menor dureza y el mecanismo de desgaste de deformación plástica presentado en el momento del ensayo de pin on disk, debido a que el material se acumuló alrededor de la pista de desgaste sin ser desprendido en forma de residuo, como se aprecia en la Fig. 5, lo que asegurará problemas en el momento de llevar a cabo un proceso de mecanizado. No obstante, esta pérdida de masa se considera despreciable. 


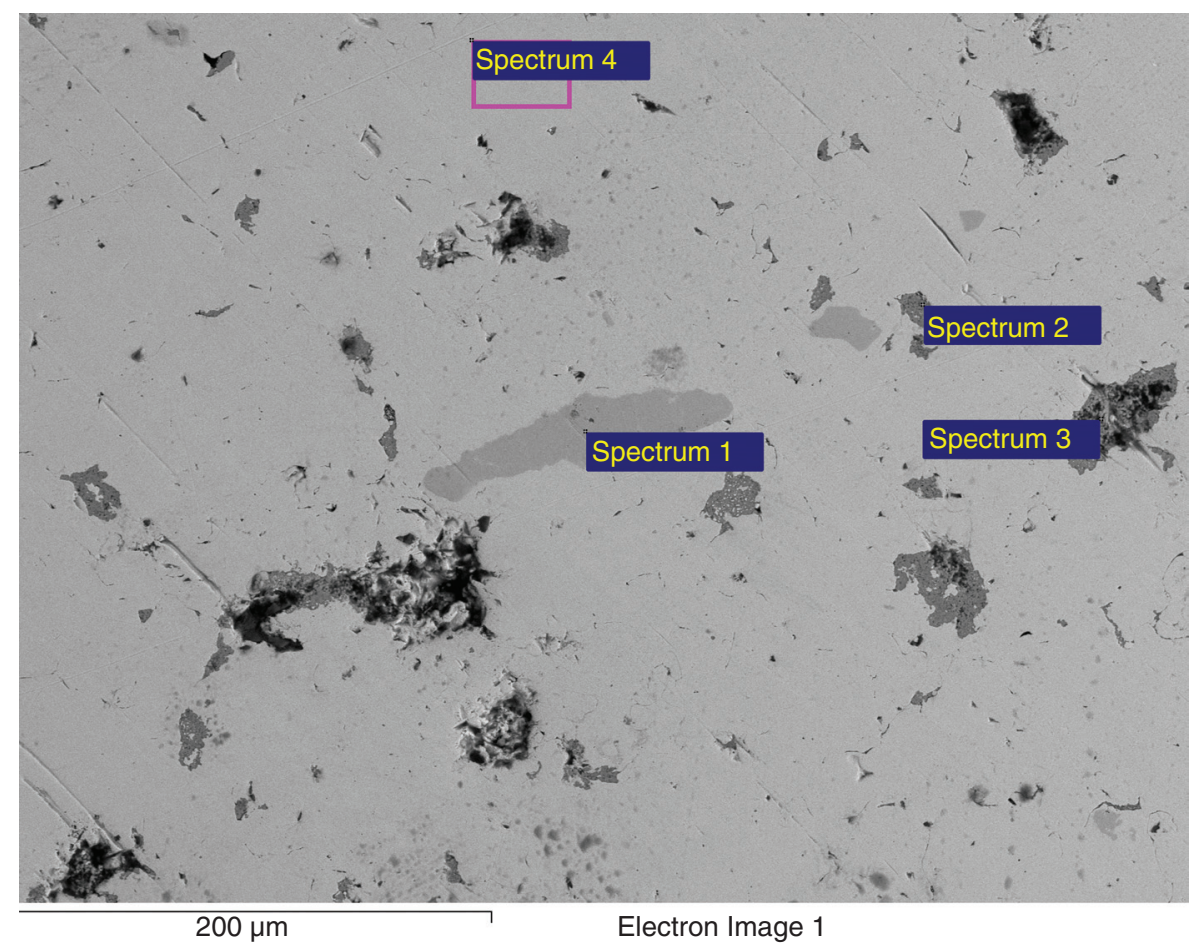

\begin{tabular}{ccccc}
\hline Spectrum & $\mathrm{Cu}$ & $\mathrm{Zn}$ & $\mathrm{Mn}$ & $\mathrm{Fe}$ \\
\hline Spectrum 1 & 0,61 & 0 & 0 & 99,39 \\
Spectrum 2 & 5,05 & 4,54 & 66,54 & 0 \\
Spectrum 3 & 2,74 & 3,71 & 72,53 & 0 \\
Spectrum 4 & 66,99 & 29,09 & 0 & 0 \\
\hline
\end{tabular}

FiguRE 4. Análisis de composición química a guía de válvula fabricada por PM.

TABla 7. Coeficiente de fricción de guías de válvulas

\begin{tabular}{lccccc}
\hline \multicolumn{5}{c}{ Coeficiente de Fricción $(\mu \mathrm{m})$} \\
\hline Zona & $\mathbf{L 4 7}$ & $\mathbf{L 4 9}$ & $\begin{array}{c}\text { L47 } \\
\text { Reprensada }\end{array}$ & $\begin{array}{c}\text { L49 } \\
\text { Reprensada }\end{array}$ & Comercial \\
\hline A & 0,47 & 0,28 & 0,34 & 0,25 & 0,15 \\
B & 0,17 & 0,23 & 0,28 & 0,16 & - \\
C & 0,17 & 0,20 & 0,32 & 0,37 & - \\
\hline
\end{tabular}

TABLA 8. Valores de pérdida de masa de GV

\begin{tabular}{lccccc}
\hline \multicolumn{5}{c}{ Pérdida de masa (g) } \\
\hline $\mathbf{Z}$ & $\mathbf{L 4 7}$ & L47 Reprensada & $\mathbf{L 4 9}$ & L49 Reprensada & C \\
\hline A & $1,8.10^{-3}$ & $3,3.10^{-4}$ & $6,7.10^{-5}$ & $4,9.10^{-3}$ & $9,7.10^{-3}$ \\
B & $4,8.10^{-3}$ & $3,7.10^{-4}$ & $6,7.10^{-5}$ & $2.10^{-4}$ & - \\
C & $5,7.10^{-4}$ & $7,4.10^{-3}$ & $6,6.10^{-4}$ & $9,1.10^{-3}$ & - \\
\hline
\end{tabular}




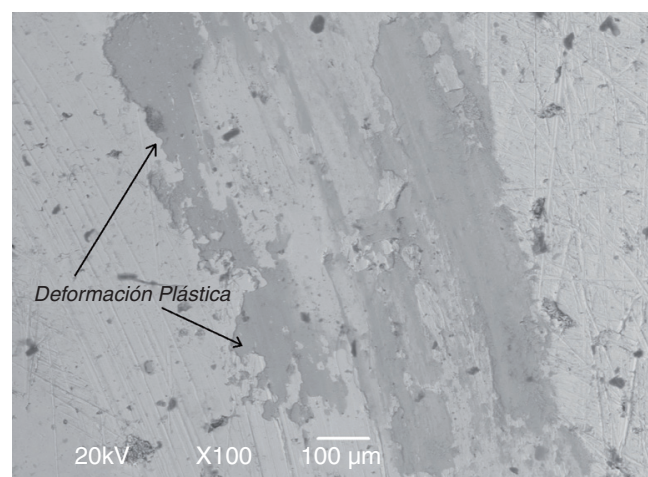

Figure 5. Mecanismo de desgaste presentado en prueba de pin on disk.

\section{CONCLUSIONES}

- Se evidenció que las GV con proceso de reprensado presentaron un incremento de dureza en comparación con GV fabricadas solo con proceso de sinterizado, sin embargo, con este proceso adicional no se consigue un comportamiento homogéneo de esta propiedad, debido a que los punzones de la prensa no ejercen igual presión sobre la guía. Esta diferencia de durezas posiblemente generará una aptitud mecánica baja.

- Los valores de dureza Vickers presentaron gran variación entre cada zona debido a la baja homogeneidad de la composición química base, presente en las GV fabricadas por PM, debido a que la temperatura de sinterización produce una pérdida de $\mathrm{Zn}$, presentando zonas sobresaturadas de $\mathrm{Cu}$, de acuerdo al análisis de composición química.

- Los valores de coeficiente de fricción en las GV fabricadas por PM y las comerciales fueron inferiores al valor estándar de coeficiente de fricción de un contacto acero-latón. Este resultado es interesante para el proceso de producción industrial de las guías de válvulas. Esta propiedad no fue homogénea en las GV por proceso de PM, lo que podrá generar desgaste e incremento de temperatura no uniforme.

- Las GV fabricadas por proceso de PM presentaron pérdida de masa despreciable, lo que indica alta resistencia al desgaste, siendo esto importante para que en funcionamiento no pierdan masa al hacer contacto con el vástago de la válvula. Esta baja de pérdida de masa presentada en las GV está relacionada con la baja dureza y con el mecanismo de desgaste de deformación plástica presentado en la prueba de pin on disk.

- Con base en los resultados anteriores, se puede concluir que las GV fabricadas por proceso de PM en empresa de la ciudad de Cali, presentan valores inferiores a nivel mecánico-tribológico en comparación con las guías del comercio. Existe la posibilidad de mejorar en las GV su homogeneidad de densidad y composición química mediante ajustes en parámetros en el proceso de fabricación.

\section{RECOMENDACIONES}

- Evaluar los tiempos en el proceso de mezclado del material en polvo para obtener una composición química más homogénea.

- Revaluar los parámetros del proceso de reprensado, donde los punzones de la prensa realicen la misma carga y actúen al mismo tiempo; para lograr mayor homogeneidad en su densidad.

- Revaluar la temperatura empleada en el proceso de sinterizado para evitar pérdidas de $\mathrm{Zn}$ y considerar el aumento de la presión de compactación o tiempo de sinterización con el objetivo de disminuir aún más la porosidad, comprobado por Jabur (2013).

\section{REFERENCIAS}

Abdizadeh, H., Ebrahimifard, R., Baghchesara, M.A. (2014). Investigation of microstructure and mechanical properties of nano $\mathrm{MgO}$ reinforced $\mathrm{Al}$ composites manufactured by stir casting and powder metallurgy methods: A comparative study. Compos. Parte B-Eng. 56, 217-221. http:// dx.doi.org/10.1016/j.compositesb.2013.08.023

ASTM G99-05 (2000). Standard Test Method for Wear Testing with Pin-on-Disk Apparatus, ASTM International, West Conshohocken, PA, USA.

ASTM E10-12 (2012). Standard Test Method for Brinell Hardness of Metallic Materials, ASTM International, West Conshohocken, PA, USA.

ASTM E92-82 (2003). Standard Test Method for Vickers Hardness of Metallic Materials, ASTM International, West Conshohocken, PA, USA.

De Garmo, E.P., Black, J.T., Kohser, R.A. (2002). Pulvimetalurgia, en Materiales y procesos de fabricación. Vol. I, $2^{\text {da }}$ Edition, Reverté S.A, Barcelona, pp. 371-386.

Dietsche, K. (2005). Manual de la técnica del automóvil. Motor de émbolo de combustión interna, $4^{\text {ta }}$ Edition, Bosch, Alemania, pp. 451-469.

Fujiki, A. (2001). Present state and future prospects of powder metallurgy parts for automotive applications. Mater. Chem. Phys. 67, 298-306. http://dx.doi.org/10.1016/ S0254-0584(00)00455-7.

Gonzales Calleja, D. (2015). Motores térmicos y sus sistemas auxiliares. Elementos Constructivos, $2^{\mathrm{da}}$ Edition, Ediciones Paraninfo S.A., Madrid, pp.105-110.

Groover, P. (1997). Fundamentos de Manufactura Moderna: Materiales, Procesos y Sistemas. Metalurgia de Polvos, $1^{\text {ra }}$ Edition, Prentice Hall, Naucalpán de Juárez, pp. 393-415.

Jabur, A.S. (2013). Effect of powder metallurgy conditions on the properties of porous bronze. Powder Technology 237, 477-483. http://dx.doi.org/10.1016/j.powtec.2012.12.027.

Jang, G.B., Hury, M.D., Kang, S.A. (2000). A study on the development of a substitution process by powder metallurgy in automobile parts. J. Mater. Process. Technol. 100 (1-3), 110 115. http://dx.doi.org/10.1016/S0924-0136(00)00426-X.

Keraghel, F., Loucif, K., Delplacke, M.P. (2011). Study of bronze porous alloy $\mathrm{Cu}-\mathrm{Sn}$ Worked out by metallurgy of the powder. Phys. Procedia 21, 152-158. http://dx.doi. org/10.1016/j.phpro.2011.10.023. 
Martin, F., García, C., Blanco, Y. (2015). Influence of residual porosity in the dry and lubricated sliding wear of a powder metallurgy austenitic stainless steel. Wear 328-329, 1-7. http://dx.doi.org/10.1016/j.wear.2015.01. 025

Mott, R.L. (2006). Diseño de elementos de máquinas. Cap. Materiales en el diseño mecánico, $4^{\text {ta }}$ Edition, Pearson Educación, Naucalpán de Juárez, p. 56.
ProExport (2102). Incentivos del sector automotriz. Colombia. (Acceso febrero 2017). http://inviertaencolombia.com. co/publicaciones/incentivos-del-sector-automotriz-2016. html.

Schmid, S.R., Kalpakjian, S. (2002). Manufactura, Ingeniería y Tecnología. Procesamiento de metales en polvo, cerámicos, vidrio y superconductores, $4^{\text {ta }}$ Edition, Prentice Hall, Naucalpan de Juárez, pp. 440-460. 